\title{
40 \\ CONTINUOUS MANAGEMENT OF PROFESSIONAL VIRTUAL COMMUNITY INHERITANCE BASED ON THE ADAPTATION OF SOCIAL PROTOCOLS
}

\author{
Willy Picard \\ Department of Information Technology \\ The Poznan University of Economics \\ ul. Mansfelda 4 \\ 60-854 Poznań, POLAND \\ picard@kti.ae.poznan.pl
}

Support for human-to-human interactions over a network is still insufficient, particularly for professional virtual communities (PVC). Among other limitations, adaptation and learning-by-experience capabilities of humans are not taken into account in existing models for collaboration processes in PVC. This paper presents a model for adaptive human collaboration. A key element of this model is the use of negotiation for adaptation of social protocols modeling processes. A second contribution is the proposition of various adaptation propagation strategies as means for continuous management of the PVC inheritance.

\section{INTRODUCTION}

Enterprises are constantly increasing their efforts in order to improve their business processes. A main reason for this may be the fact that enterprises are exposed to a highly competitive global market. Among the most visible actions associated with this effort towards better support for better business processes, one may distinguish the current research work concerning Web services and associated standards: highlevel languages such as BPEL or WS-Coordination take the service concept one step further by providing a method of defining and supporting workflows and business processes.

However, it should be noticed that most of these actions are directed towards interoperable machine-to-machine interactions over a network. Support for humanto-human interactions over a network is still insufficient and more research has to be done to provide both theoretical and practical knowledge to this field.

Among various reasons for the weak support for human-to-human interactions, one may distinguish the following three reasons: first, many social elements are involved in the interaction among humans. An example of such a social element may be the role played by humans during their interactions. Social elements are usually difficult to model, i.e. integrating non-verbal communication to collaboration models. Therefore, their integration to a model of interaction between humans is not easy. A second reason is the adaptation capabilities of humans which are not only far more advanced than adaptation capabilities of software entities, but also not taken into account in existing models for collaboration processes. A third reason is the learning-by-experience capabilities of humans, i.e. the capabilities to extract knowhow and knowledge from previous experience and reuse it in similar situations.

Picard, W., 2007, in IFIP International Federation for Information Processing, Volume 243, Establishing the Foundation of Collaborative Networks; cds. Camarinha-Matos, L., Afsarmanesh, H., Novais, P., Analide, C.; (Boston: Springer), pp. 381-388. 
The insufficient support for human-to-human interactions over a network is a strong limitation for a wide adoption of professional virtual communities (PVCs). As mentioned in (Camarinha-Matos et al., 2005), "professional virtual community represents the combination of concepts of virtual community and professional community. Virtual communities are defined as social systems of networks of individuals, who use computer technologies to mediate their relationships. Professional communities provide environments for professionals to share the body of knowledge of their professions [...]". According to Chituc and Azevedo (2005), little attention has been paid to the social perspective on Collaborative Networks (CN) business environment, including obviously professional virtual communities in which social aspects are of high importance.

This paper is an attempt to provide a model for human-to-human interactions within professional virtual communities. The proposed model addresses, at least to some extent, the three characteristics of the interactions between humans. It should however been kept in mind that the results presented here are a work in progress and therefore they are not claimed to be neither sufficient nor exhaustive.

The rest of this paper is organized as follows. In section 2, the concept of social protocol, used to model collaboration processes, is presented. Section 3 then expands on adaptation of social protocols. Next, the continuous management of PVC-inheritance based on adaptation propagation strategies is discussed. Finally, section 5 concludes this paper.

\section{STRUCTURING COLLABORATION IN PVCS}

\subsection{PVCs as Heterogeneous and Dynamic Environments}

As defined by Ekholm and Fridqvist (1996), "a human sociosystem has a composition of human individuals, its structure is the social behaviour repertoire, i.e. interaction among human individuals". The sociosystem of professional virtual communities is highly heterogeneous and dynamic.

The heterogeneity of PVCs exists at various levels of granularity within PVCs. At a high level, a PVC consists usually of many different "sub-communities". Each sub-community is different from other coexisting in the same PVC sub-communities in terms of goals, intentions, knowledge, processes, members, etc. At a lower level, one may notice that the structure of a sub-community is usually complex and heterogeneous. The roles played by the sub-community members, their skills, their competences are usually presenting a high level of diversity.

Similarly to the heterogeneity of PVCs, the dynamics of PVCs exists at various levels of granularity within PVCs. At a high level, the set of sub-communities that the PVC consists of evolves in time: new sub-communities are created to answer new needs and opportunities, unnecessary sub-communities are dissolved, existing sub-communities change as new members enter and leave the community, etc. The dynamics of PVCs may hardly, not to say cannot, be foreseen at design time, as changes of a given PVC are naturally related to changes in its business environment (which is usually not a deterministic system). At a lower level, the structure of a sub-community is evolving in time: some members may have job promotion, the skills of the members are usually evolving (improve) in time. Additionally, members 
of a given sub-community may face new situations implying the development of new solutions, new ways of collaboration, etc.

\subsection{Modeling Group Interactions with Social Protocols}

Support for human-to-human collaboration in PVCs should obviously take into account the characteristics of PVCs as sociosystems presented in the former subsection, i.e. heterogeneity and dynamics.

A first model for group interactions within a PVC has been presented in (Picard, 2005). The proposed model is based on the concept of social protocol. Social protocols model collaboration at a group level. The interactions of collaborators are captured by social protocols. Interactions are strongly related to social aspects, such as the role played by collaborators. The proposed model integrates some of these social aspects, which may explain the choice of the term "social protocols". Heterogeneity of PVCs at the sub-community level is then at least partially addressed by the social protocol approach.

A social protocol aims at modeling a set of collaboration processes, in the same way as a class models a set of objects in object-oriented programming. In other words, a social protocol may be seen as a model which instances are collaboration processes. Within a given PVC, various social protocols may be used to control interactions within different sub- communities, addressing at least partially the high level heterogeneity of PVCs.

Formally, a social protocol $p$ is a finite state machine consisting of $\left\{S_{p}, S_{p}^{\text {start }}\right.$, $\left.S_{p}{ }^{\text {end }}, T p\right\}$ where $S_{p}$ is the set of states, $S_{p}^{\text {start }} \subset S$ is the set of starting states, $S_{p}{ }^{\text {end }} \subset S$ is the set of ending states, $S_{p}^{\text {start }} \cap S_{p}{ }^{\text {end }}=\varnothing, T_{p}$ is the set of transitions from states to states.

In a social protocol, collaborators - as a group -move from state to state via the transitions. A transition may be triggered only by a collaborator labeled with the appropriate role. A transition is associated with the execution of an action. Execution of an action means the execution of remote code. SOAP or CORBA are examples of technologies that may be used to such remote code executions.

A set of group actions have been identified to support group dynamics, i.e. the dynamics of PVCs at a high level. A group action is a special action that may be executed to modify the set of sub-communities that the PVC consists of. A group action may for instance allows a collaborator to split a group in two or more groups, or to merge two or more groups into a single group. Group dynamics may be modeled by a set of group actions.

A formal definition of the proposed model has been already presented in (Picard, 2006a), while an algorithm for structural validation of social protocols has been presented in (Picard, 2007).

\section{ADAPTIVE SOCIAL PROTOCOLS}

Social protocols address heterogeneity of PVCs at both high and low level, and dynamics at high level. However, the need for support for dynamics of PVCs is still only partially addressed at the sub-community level. 


\subsection{Run-time vs. Design-Time Adaptation}

In the workflow management literature, information required to model and control a collaboration process has been classified according to various perspectives.

In (van der Aalst et al., 2003), five perspectives have been presented:

$>$ the functional perspective focuses on activities to be performed,

$>$ the process perspective focuses on the execution conditions for activities,

$>$ the organization perspective focuses on the organizational structure of the population that may potentially execute activities,

$>$ the information perspective focuses on data flow among tasks,

$>$ the operation perspective focuses on elementary operations performed by applications and resources.

A sixth perspective has been added in (Daoudi and Nurcan, 2003): the intentional perspective focuses on goals and strategies related to a given process. One may easily notice that all six perspectives presented above focus on elements that evolve in time.

In typical workflow management systems, two parts may be distinguished: a design-time part allows for definition of workflow schemas while the run-time part is responsible for execution of workflow instances. A main limitation of typical workflow management systems is the fact that once a workflow schema has been instantiated, the execution of the workflow instance must stick to the workflow schema till the end of the workflow instance execution. This limitation is not an issue if the lifespan of workflow instances is short in comparison with the time interval between two requests for changes of the workflow schema. When the lifespan of workflow instances is long in comparison with the time interval between two requests for changes of the workflow schema, a high number of workflow instances has to be executed with an "incorrect" workflow schema (i.e. that does not take into account required changes) or cancelled. As a consequence, typical workflow management systems are not flexible enough to support collaborative processes in two cases: highly dynamic, competitive markets/environments and long lasting collaboration processes.

In the case of highly dynamic, competitive markets/environments or long lasting collaboration processes, there is a strong need for the possibility to modify a workflow instance at run-time. Such modifications are usually needed to deal with situations which have not been foreseen nor modeled in the associated workflow schema. Social protocol adaptation refers to the possibility to modify a running social protocol instance to new situations which have not been foreseen and modeled in the associated social protocol.

\subsection{Negotiation-based Adaptation}

While social protocols support, at least to some extent, the integration of some social elements (such as roles) to models of interactions among humans, the adaptation capabilities of humans are not taken into account into social protocols. There is however the need to provide adaptation mechanisms to social protocols. Indeed, interactions among humans are often a context-aware activity. In this paper, contextawareness refers to the capabilities of applications to provide relevant services to their users by sensing and exploring the users' context (Dey et al., 2001; Dockhorn 
et al., 2005). Context is defined as a "collection of interrelated conditions in which something exists or occurs" (Dockhorn et al., 2005). The users' context often consists of a collection of conditions, such as, e.g., the users' location, environmental aspects (temperature, light intensity, etc.) and activities (Chen et al., 2003). The users' context may change dynamically, and, therefore, a basic requirement for a contextaware system is its ability to sense context and to react to context changes.

In (Picard, 2006b), negotiations have been proposed as a method for adaptation of social protocols. The idea of negotiation of social protocol has been presented as "an attempt to weaken constraints usually limiting the interaction between collaborators, so that the adaptation capabilities of humans may be integrate in the life of a social protocol". The idea of using negotiations as an adaptation mean for social protocols comes from the fact that social protocols rule the interactions of all collaborators in a given group. Therefore each modification of the social protocol may influence all collaborators. As a consequence, the decision to modify a social protocol should be consulted and approved by many collaborators. Negotiations are a classical way to make collaborative decision and to reach an agreement in situations where expectations and goals of collaborators may be in conflict.

\section{ADAPATION OF SOCIAL PROTOCOLS IN PVCS}

In the context of PVCs, adaptation leads to support for dynamics of collaboration processes at the group level. Additionally, decisions taken during adaptation of social protocols may be reused by other groups facing similar problems.

\subsection{Adaptation Propagation Strategies}

Adaptation of a social protocol in a given group leads to the creation of a new version of the social protocol ruling collaboration within this group. Let assume that the adaptation of a given social protocol $\mathrm{P} 1$ in a given group $\mathrm{G}$ leads to the creation of a new social protocol Pl'. In the context of a PVC, various strategies may be used to manage the change caused by the adaptation of a social protocol:

$>$ Local adaptation strategy: Other groups ruled by the social protocol $P$ are not affected by the adaptation and are still ruled by $P$. The social protocol $P$ ' is only used by group $G$ and is not available for future groups.

$>$ Global propagation strategy: Other groups ruled by the social protocol $P$ are not affected by the adaptation and are still ruled by $P$. The social protocol $P$ ' is used by group $G$ and is available for future groups.

$>$ Instant propagation strategy: Other groups ruled by the social protocol $P$ are affected by the adaptation, as they are now ruled by $P^{\prime}$. The social protocol $P^{\prime}$ replaces $P$ in the whole $\mathrm{PVC}$.

It should be noticed that the instant propagation strategy may not always be used as the changes provided by the adaptation of the social protocol may be in conflict with the current state of some collaboration processes.

\subsection{Adaptation Propagation in a VO-Inheritance Management Perspective}

The concept of virtual organization inheritance (VO-I) has been defined in (Loss et al., 2006a) as "the set of information and knowledge accumulated from past and 
current VOs along their entire life cycle. Virtual organization inheritance management (VO-I-M) corresponds to the VO activity that manages what has been inherited about given VOs, usually supported by computer systems".

a)

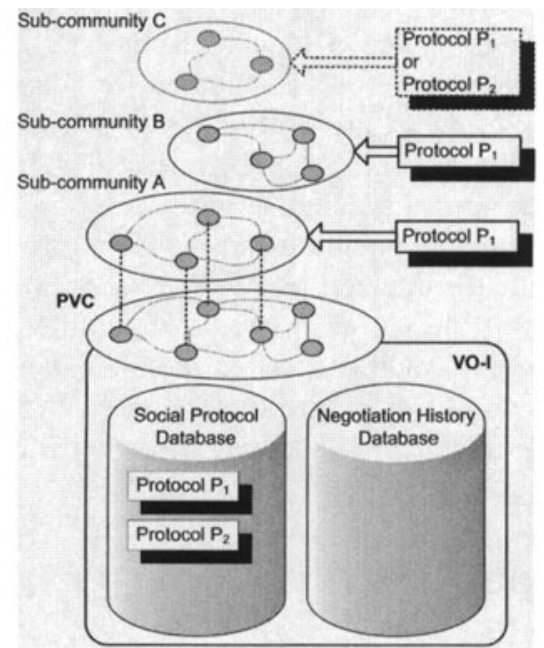

c)

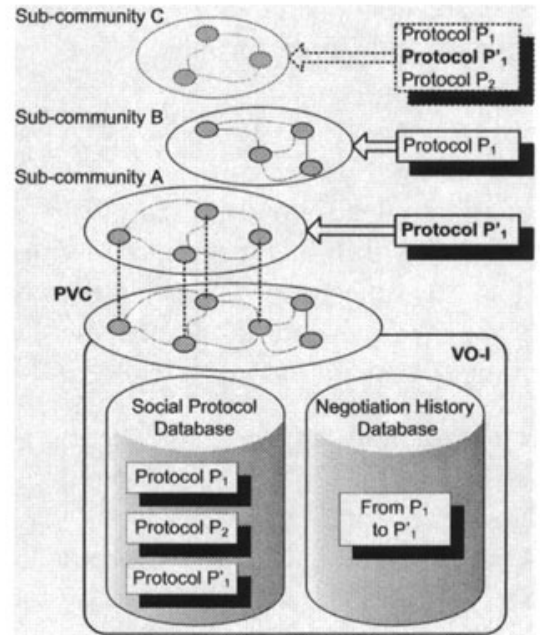

b)

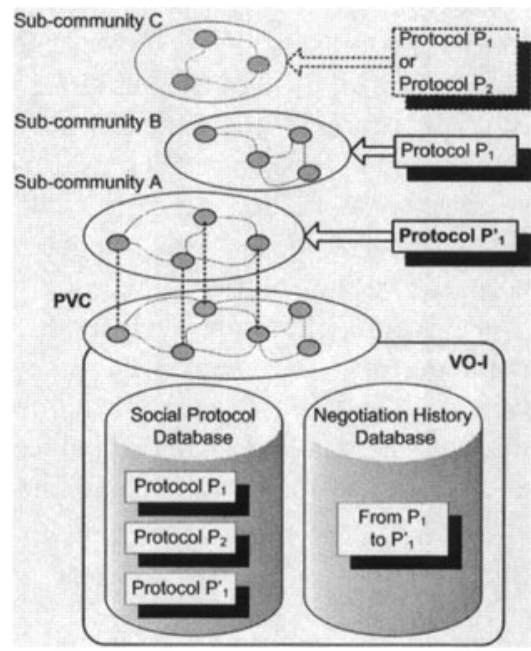

d)

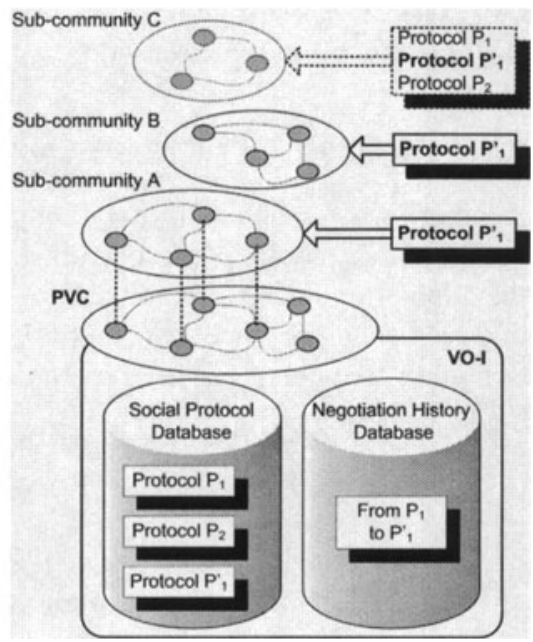

Figure 1 - VO-I a) before adaptation b) with a local adaptation strategy c) with a global propagation strategy d) with an instant propagation strategy

In a VO-I-M perspective, adaptation of social protocols may be seen as part of the VO-I, as presented in Figure 1. In the PVC presented in Figure 1a), two protocols are available - P1 and P2 - and two sub-communities - A and B - are ruled by $\mathrm{P} 1$. A new sub-community $\mathrm{C}$ may be created with either protocol $\mathrm{P} 1$ or protocol $\mathrm{P} 2$. It is then assumed that the sub-community $\mathrm{A}$ has adapted the protocol P1, which leads to the protocol P'1. Figure 1b) (respectively 1c) and 1d)) illustrates the state of the PVCs after the adaptation in the case of a local adaptation strategy (respectively global and instant adaptation strategy). In Figure 1b), the newly created protocol $P{ }_{I}$ 
rules the sub-community $A$ but is not available to sub-communities $B$ and $C$. In Figure 1c), the newly created protocol is available to the new sub-community $C$ but the sub-community $B$ is still ruled by $P_{l}$. In Figure $1 \mathrm{~d}$ ), $P^{\prime}{ }_{l}$ is available to the new sub-community $C$ and the sub-community $B$ is now ruled by $P^{\prime}{ }_{l}$.

The newly created social protocol $P^{\prime}{ }_{l}$ embeds knowledge about an alternative way to collaborate. The social protocol $P^{\prime}$, models the additional knowledge and expertise which have been required to react to situations which have not been unforeseen nor modeled in the social protocol $P_{l}$. Information about the negotiation process that leads from $P_{1}$ to $P_{1}^{\prime}$ is available from the negotiation history database, as presented in Figure 1. One should notice that these knowledge and expertise should not necessarily been directly reused, but could be used for consultation about what has happened in similar cases and the solution found. Obviously, privacy should be taken into account. Collaborators that negotiated the social protocol $P_{I}$ should explicitly agree to publish negotiation-related information before such information is available to other sub-communities.

The global propagation strategy would allow collaborators of sub-communities to consult and eventually reuse the VO-I of sub-communities in which a social protocol has been adapted. The instant propagation strategy would enforce the reuse of newly-created knowledge by other sub-communities in a normative way: the adapted social protocol "overwrites" the original social protocol.

Finally, the proposed adaptation propagation strategies provide means for continuous VO-I-M. A classical issue in VO-I-M is the frequency of VO-I capturing. A briefing-debriefing technique has been presented by Loss et al. (2006b), proposing to capture VO-I by comparing the results of two interview meetings: usually the first interview meeting takes place before the VO is created, while the second one (the debriefing) takes place after VO dissolution or metamorphosis. The briefing-debriefing technique may be used "to double-check the plans, fine tune the assignments of tasks, rehearsal the actions and also to exchange lessons learned, evaluate the actions against the plans and to register explicitly the knowledge acquired, respectively". Therefore, the briefing-debriefing technique may capture more elements of the VO-I, than just those related to social protocols. On the second hand, information about adaptation of a social protocol would be captured by the briefing-debriefing technique during the debriefing session, while the adaptation propagation strategies make information about adaptation of a social protocol accessible by other VOs just after the adaptation. Therefore, propagation strategies may enable continuous VO-I-M of social protocols, while the briefing-debriefing technique is less agile but may capture more elements of the VO-I.

\section{CONCLUSIONS}

The introduction of adaptation of social protocols and adaptation propagation strategies to provide computer support to management of PVC-inheritance related to collaboration processes. To our best knowledge, it is the first attempt to support continuous management of VO-inheritance, even if the proposed solution is limited to PVC-inheritance elements related to collaboration processes.

The main innovations presented in this paper are 1) the rational for adaptation of social protocols in PVCs as heterogeneous and dynamic sociosystems, 2) three strategies for adaptation propagation, 3) the proposition of adaptation of social 
protocols and adaptation propagation as means for continuous management of PVC inheritance. Among future works, a formal model of propagation strategies presented in this paper should be established and validated by experiments.

\section{REFERENCES}

1. van der Aalst, W.M.P., Weske, M. and Wirtz, G. (2003) Advanced topics in workflow management: Issues, requirements, and solutions. J. of Integrated Design and Process Science, 7(3), pp. 49-77

2. Camarinha-Matos, L.M., Afsarmanesh, H. and Ollus, M. (2005) ECOLEAD: A Holistic Approach to Creation and Management of Dynamic Virtual Organizations. In Collaborative Networks and their Breeding Environments, Proc. of the 6th IFIP Working Conf. on Virtual Enterprises (PRO-VE 2005), Valencia, Spain, Sept. 26-28, 2005, Springer, pp. 3-16

3. Chen, H., Finin, T. and Joshi, A. (2003) An Ontology for Context-Aware Pervasive Computing Environments. Knowledge Engineering Review, Special Issue on Ontologies for Distributed Systems, 18(3) Cambridge University Press, pp. 197-207

4. Chituc, C.M., and Azevedo, A.L. (2005) Multi-Perspective Challenges on Collaborative Networks Business Environments. In Collaborative Networks and their Breeding Environments, Proceedings of the 6th IFIP Working Conf. on Virtual Enterprises (PRO-VE 2005), Valencia, Spain, Sept. 26-28, 2005, Springer, pp. 25-32

5. Daoudi, F. and Nurcan, S. (2007) A benchmarking framework for methods to design flexible business processes. Special Issue on Design for Flexibility of the "Software Process: Improvement and Practice Journal", 12(1), pp. 51-63

6. Dey, A.K., Salber, D. and Abowd, G. D. (2001) A Conceptual Framework and a Toolkit for Supporting the Rapid Prototyping of Context-Aware Applications. Human-Computer Interaction, 16(2-4), pp. 97-166

7. Dockhorn Costa, P., Ferreira Pires, L. and van Sinderen, M. (2005) Designing a Configurable Services Platform for Mobile Context-Aware Applications. Int. J. of Pervasive Computing and Communications (JPCC), 1(1), pp. 27-37

8. Ekholm, A. and Fridqvist, S. (1996) Modelling of user organisations, buildings and spaces for the design process. In Construction on the Information Highway, Proceedings from the CIB W78 Workshop, Bled, Slovenia, June 10-12, 1996

9. Loss, L., Pereira-Klen, A.A. and Rabelo, R.J. (2006a) Knowledge Management Based Approach for Virtual Organization Inheritance. In Network-centric Collaboration and Supporting Frameworks, Proc. of the 7th IFIP Working Conf. on Virtual Enterprises (PRO-VE 2006), Helsinki, Finland, Sept. 2006. Springer, pp. 285-294

10. Loss, L., Rabelo, R.J. and Pereira-Klen, A.A. (2006b) Virtual Organization Management: An Approach Based on Inheritance Information. In: Global Conference on Sustainable Product Development and Life Cycle Engineering, São Carlos, SP, Brazil, Editora Suprema

11. Picard, W. (2007) An Algebraic Algorithm for Structural Validation of Social Protocols. In Lecture Notes in Computer Science, 4439, Springer, pp. 570-583

12. Picard, W. (2006a) Adaptive Collaboration in Professional Virtual Communities via Negotiations of Social Protocols. In Network-centric Collaboration and Supporting Frameworks, Proc. of the 7th IFIP Working Conf. on Virtual Enterprises (PRO-VE 2006), Helsinki, Finland, Sept. 2006. Springer, pp. 353-360

13. Picard, W. (2006b) Adaptive Human-to-Human Collaboration via Negotiations of Social Protocols". In Technologies for Business Information Systems, Proc. of the 9th Int. Conf. on Business Information Systems, Klagenfurt, Austria, May 31 - June 2, 2006, Springer Verlag, pp. 193-203

14. Picard, W. (2005) Modeling Structured Non-monolithic Collaboration Processes. In Collaborative Networks and their Breeding Environments, Proc. of the 6th IFIP Working Conf. on Virtual Enterprises (PRO-VE 2005), Valencia, Spain, Sept. 26-28, 2005, Springer, pp. 379-386 